\title{
UMA EXPLORAÇÃO DA ESPECIFICIDADE E INTERDISCIPLINARIDADE METODOLÓGICA EM PSICOLOGIA AMBIENTAL
}

\author{
Emilio Moyano Díaz ${ }^{1}$ \\ Universidade do Chile
}

\begin{abstract}
Este artigo propõe uma exploração e uma explicitação da especificidade $e$ da interdisciplinaridade metodológica em Psicologia Ambiental, apoiando-se em tipologias existentes referentes aos tipos de problemas, de investigações e de enfoques metodológicos. Conclui por um pluralismo metodológico, os métodos sendo produtos úteis, mesmo que culturais, para buscar respostas a um problema, se considerados quanto à sua eficácia e à sua eficiência empíricas. A intervenção ambiental é, por sua vez, marcada pela intervenção social.
\end{abstract}

Descritores: Pesquisa interdisciplinar. Metodologia. Psicologia Ambiental.

\begin{abstract}
Asicologia Ambiental é uma integração da ciência básica - para expliAcar a relação entre comportamento, experiência humana e ambientes físicos (e sociais) - com a ciência aplicada - para otimizar a relação entre os seres humanos e seus ambientes. Este último é compartilhado também com o urbanismo e a arquitetura, disciplinas da habitabilidade.
\end{abstract}

A especificidade da Psicologia Ambiental com respeito a outras disciplinas ambientais está em seu objeto de estudo que considera o comporta-

1 Professor das Universidades do Chile, Católica do Chile e de Santiago; especialista em Metodologia de Avaliação residencial e coordenador do Programa de Especialização em Moradia Social da Faculdade de Arquitetura e Urbanismo da Universidade do Chile. Endereço eletrônico: emoyano@lauca.usach.cl 
mento humano como variável a explicar, ou dependente de condições físico ambientais e, em menor grau, como variável explicativa de eventuais mudanças ambientais, ou variáveis independentes.

O termo metodologia designa o caminho ou estilo de enfrentamento da realidade, de como formulamos os problemas e buscamos respostas. De modo mais preciso e restritivo, o método científico é um conjunto de prescrições falseáveis que serve para formular adequadamente perguntas, planejar investigações e experimentos e interpretar os seus resultados (Bunge, 1992).

É evidente que os métodos (e técnicas) em ciência não são algo "naturalmente dado" mas são criações ou instrumentos culturais construídos para certos propósitos - resolver perguntas e problemas - em um momento determinado (Moyano Díaz, 1994b).

Qual é o tipo de problema ou pergunta de investigação em Psicologia Ambiental? Dentre as várias classificações de problemas, existe uma que distingue quatro tipos: conceituais, metodológicos, empíricos e valorativos (Sierra Bravo, 1987). De maneira um pouco mais restrita ou sintética, Bunge e Ardila (1988) distinguem, para a Psicologia, três tipos de problemas que consideram típicos: empíricos (qual é o valor de X?: medição), teóricos (porque $\mathrm{X}$ tem o valor de $\mathrm{x}$ ?: explicação) e práticos (como modificar o valor de $X$ ?).

Quanto aos tipos de investigação, uma classificação de ampla aceitação é aquela baseada no critério de seu alcance explicativo, de quatro tipos: exploratória, descritiva, correlacional e explicativa (Hernández, Fernández, \& Baptista, 1993). Devido à amplitude do método científico e à criatividade dos pesquisadores, existe uma variedade de formas de investigar que ocupam um lugar importantíssimo no fazer científico e, muito especialmente, no profissional. Com um enfoque com critérios múltiplos, selecionamos um que distingue sete tipos de pesquisas, analisados a partir de sete critérios classificatórios (colunas) (De Ketele, 1984; em De Ketele \& Roegiers, 1993). (Quadro 1). 
Quadro 1. Classificação de Tipos de Investigação (de Ketele, 1984)

\begin{tabular}{|c|c|c|c|}
\hline $\begin{array}{l}\text { TIPOS DE INVESTI- } \\
\text { GAÇÃO }\end{array}$ & $\begin{array}{l}\text { CRITERIO } 1 \\
\text { Investigação prioritari- } \\
\text { amente orientada a }\end{array}$ & $\begin{array}{l}\text { CRITERIO } 2 \\
\text { Necessidade de prever e } \\
\text { controlar }\end{array}$ & $\begin{array}{l}\text { CRITERIO } 3 \\
\text { Experiência estrita de } \\
\text { medidas válidas e con- } \\
\text { fiáveis. }\end{array}$ \\
\hline $\begin{array}{l}1 . \\
\text { IC: Investigação cientí- } \\
\text { fica. } \\
\text { ICF e CL: Investigação } \\
\text { científica básica e de } \\
\text { laboratório. } \\
\text { IC: Investigação cientí- } \\
\text { fica de campo. }\end{array}$ & $\begin{array}{l}\text { Conclusões: } \\
\text { Leis científicas fundamen- } \\
\text { tais } \\
\text { Leis científicas aplicáveis. }\end{array}$ & $\begin{array}{l}\text { Sim. } \\
\text { Dispositivos experimentais, } \\
\text { quase-experimentais, corre- } \\
\text { lacionais, segundo os casos. } \\
\text { Idem. }\end{array}$ & Sim. \\
\hline $\begin{array}{l}2 . \\
\text { ID OIT (OIA): } \\
\text { Investigação de de- } \\
\text { senvolvimento ou tec- } \\
\text { nológica (aplicada). }\end{array}$ & $\begin{array}{l}\text { Decisões generalizáveis. } \\
\text { Leis e regras tecnológi- } \\
\text { cas, instrumentos materi- } \\
\text { ais (visíveis e generalizá- } \\
\text { veis). }\end{array}$ & $\begin{array}{l}\text { Sim. } \\
\text { Dispositivos experimentais, } \\
\text { quasi experimentais, corre- } \\
\text { lacionais segundo os casos. }\end{array}$ & Sim. \\
\hline $\begin{array}{l}3 . \\
\text { IE IO: } \\
\text { Investigação avaliativa } \\
\text { u operacional. }\end{array}$ & $\begin{array}{l}\text { Decisões científicas. } \\
\text { Estas decisões são enun- } \\
\text { ciadas pelos decisores. } \\
\text { Objetivo: } \\
\text { Realizar bem e comunicar } \\
\text { uma avaliação válida e } \\
\text { confiável para fundamen- } \\
\text { tar decisões }\end{array}$ & $\begin{array}{l}\text { Não. } \\
\text { Geralmente o dispositivo a- } \\
\text { valiativo se constrói ou ajus- } \\
\text { ta durante a Investigação se } \\
\text { se quer validade. }\end{array}$ & Sim. \\
\hline $\begin{array}{l}4 . \\
\text { IA: } \\
\text { Investigação - ação. }\end{array}$ & $\begin{array}{l}\text { Ação-Formulação } \\
\text { Ajudar aos participantes a } \\
\text { tomar consciência das de- } \\
\text { terminantes de sua ação } \\
\text { e a tomar decisões con- } \\
\text { seqüentes. }\end{array}$ & $\begin{array}{l}\text { Não. } \\
\text { As etapas da Investigação } \\
\text { estão subordinadas às rea- } \\
\text { ções dos participantes e aos } \\
\text { acontecimentos. }\end{array}$ & $\begin{array}{l}\text { Não. } \\
\text { O contexto não permite } \\
\text { com freqüência assegu- } \\
\text { rar a validez e a confiabi- } \\
\text { lidade das observações. }\end{array}$ \\
\hline $\begin{array}{l}5 . \\
\text { ICE o ITE: } \\
\text { Investigação científica } \\
\text { ou tecnológica explora- } \\
\text { tória. }\end{array}$ & $\begin{array}{l}\text { Formular hipóteses é o } \\
\text { objetivo. }\end{array}$ & Não. & $\begin{array}{l}\text { Sim-Não. } \\
\text { Sim, para as medidas já } \\
\text { conhecidas como válidas. } \\
\text { Não, para a observação } \\
\text { de fenômenos pouco ou } \\
\text { mal conhecidos. }\end{array}$ \\
\hline $\begin{array}{l}6 . \\
\text { ID: } \\
\text { Investigação descritiva. }\end{array}$ & $\begin{array}{l}\text { Leis e características } \\
\text { descritivas. } \\
\text { Objetivo principal: } \\
\text { Separar um contexto para } \\
\text { preparar outro processo } \\
\text { heurístico, avaliativo, etc. }\end{array}$ & $\begin{array}{l}\text { Sim. } \\
\text { Depois de ter explorado pis- } \\
\text { tas de descrição possíveis } \\
\text { se cria em uma } 2^{\text {a }} \text { fase um } \\
\text { dispositivo sistemático de } \\
\text { descrição. }\end{array}$ & Sim, na $2^{a}$ fase. \\
\hline $\begin{array}{l}7 . \\
\text { IE: } \\
\text { Investigação especulativa }\end{array}$ & $\begin{array}{l}\text { Construção de novos re- } \\
\text { ferentes de base. }\end{array}$ & Não. & Não. \\
\hline
\end{tabular}




\begin{tabular}{|c|c|c|c|c|}
\hline $\begin{array}{l}\text { TIPOS DE INVESTIGA } \\
\text { ÇÃO }\end{array}$ & $\begin{array}{l}\text { CRITERIO } 4 \\
\text { Capacidade ou neces- } \\
\text { sidade de generalização }\end{array}$ & $\begin{array}{l}\text { CRITERIO } 5 \\
\text { Replicabilidade }\end{array}$ & $\begin{array}{l}\text { CRITERIO } 6 \\
\text { Destinatários da } \\
\text { Investigação }\end{array}$ & $\begin{array}{l}\text { CRITERIO } 7 \\
\text { Valor prioritá- } \\
\text { rio }\end{array}$ \\
\hline $\begin{array}{l}1 . \\
\text { IC: Investigação científi- } \\
\text { ca. } \\
\text { ICF e CL: Investigação } \\
\text { científica básica e de la- } \\
\text { boratório. } \\
\text { IC: Investigação científica } \\
\text { de campo. }\end{array}$ & $\begin{array}{l}\text { Sim. } \\
\text { Aos contextos e trata- } \\
\text { mentos de laboratório. } \\
\text { Sim. } \\
\text { À categoria de contexto e } \\
\text { de tratamentos das a- } \\
\text { mostras. }\end{array}$ & $\begin{array}{l}\text { Sim. } \\
\text { A replicabilidade } \\
\text { é mais fácil no } \\
\text { laboratório. } \\
\text { Mais difícil no ter- } \\
\text { reno. }\end{array}$ & Investigadores. & $\begin{array}{l}\text { Verdade (ou } \\
\text { conhecimento). }\end{array}$ \\
\hline $\begin{array}{l}2 . \\
\text { ID OIT (OIA): } \\
\text { Investigação de desen- } \\
\text { volvimento ou tecnológi- } \\
\text { ca (aplicada). }\end{array}$ & $\begin{array}{l}\text { Sim. } \\
\text { Generalização da aplica- } \\
\text { bilidade a contextos bem } \\
\text { precisos. }\end{array}$ & Sim. & $\begin{array}{l}\text { Decisores- } \\
\text { Investigadores. }\end{array}$ & Eficácia. \\
\hline $\begin{array}{l}3 . \\
\text { IE IO: } \\
\text { Investigação avaliativa u } \\
\text { operacional. }\end{array}$ & $\begin{array}{l}\text { Não. } \\
\text { O objetivo prioritário é } \\
\text { fundamentar uma decisão } \\
\text { válida para um contexto } \\
\text { determinado e não ne- } \\
\text { cessariamente para um } \\
\text { conjunto de contextos. }\end{array}$ & $\begin{array}{l}\text { Não. é } \\
\text { Porque o contex- } \\
\text { to - específico. }\end{array}$ & $\begin{array}{l}\text { Decisores preo- } \\
\text { cupados com um } \\
\text { contexto especí- } \\
\text { fico. }\end{array}$ & Adequação. \\
\hline $\begin{array}{l}4 . \\
\text { IA: } \\
\text { Investigação - ação. }\end{array}$ & $\begin{array}{l}\text { Não. } \\
\text { A Investigação é conside- } \\
\text { rada somente no contexto } \\
\text { em que se realizou. }\end{array}$ & Não. & Atores. & Emancipação. \\
\hline $\begin{array}{l}5 . \\
\text { ICE o ITE: } \\
\text { Investigação científica ou } \\
\text { tecnológica exploratória. }\end{array}$ & Não. & $\begin{array}{l}\text { Sim, em muitos } \\
\text { casos. }\end{array}$ & Investigadores. & Criatividade. \\
\hline $\begin{array}{l}6 . \\
\text { ID: } \\
\text { Investigação descritiva. }\end{array}$ & Sim. & Sim. & $\begin{array}{l}\text { Investigadores } \\
\text { (ou avaliadores). }\end{array}$ & Objetividade. \\
\hline $\begin{array}{l}7 . \\
\text { IE: } \\
\text { Investigação especulati- } \\
\text { va }\end{array}$ & Não. & Não. & $\begin{array}{l}\text { Investigadores ou } \\
\text { público cultivado } \\
\text { de alto nível. }\end{array}$ & $\begin{array}{l}\text { Compreensão } \\
\text { prospectiva. }\end{array}$ \\
\hline
\end{tabular}

\section{4}


Pode-se observar um alto grau de diferenciação nos tipos de investigação científica. O pesquisador interessado no incremento da compreensão por meio da busca de conhecimento legal (que aponta para leis) realizará pesquisa do tipo 1 , enquanto quem estiver interessado em ajudar às pessoas a tomar consciência acerca dos determinantes de sua ação, realizará a 4, e um terceiro interessado em responder a clientes que tomarão decisões com a informação pertinente que o pesquisador proverá, a 3, etc. As pesquisas de tipo qualitativo estão representadas - embora não esgotadas em suas variantes - na fila 4, como Investigação-Ação.

Em geral, devido ao acento prático ou a orientação para a solução de problemas com que os autores em Psicologia Ambiental quiseram defini-la, e a heterodoxia que isto leva implícito, parece que as pesquisas nesta disciplina são classificáveis nos tipos 1, 3, 4, 6 e 7, da primeira coluna (Quadro 1), com um predomínio relativo da investigação descritiva (6) e da investigação avaliadora (3).

\section{O método em Psicologia Ambiental}

Altman e Rogoff (1991) analisam quatro enfoques em Psicologia - de traço, interativo, organísmico e transacional - que variam quanto a como se define o que é a psicologia. No enfoque de traço, os métodos predominantes são dispositivos que tornam possível a correlação (entre traços ou entre estes e o comportamento); nos interativos, é promovido o uso da experimentação de laboratório que, por sua vez, permite precisar condições antecedentes e conseqüentes entre variáveis. Nos enfoques organísmicos e transacionais (acento holístico) o que lhes é próprio são os métodos descritivos, observacionais de campo ou naturalísticos, ou outros procedimentos não experimentais. Não obstante estas tendências, não é inevitável que o enfoque teórico utilizado para abordar um certo problema implique, impreterivelmente, na utilização de um certo método, já que seria muito limitador para o desenvolvimento e inovação da ciência. 
Em geral, nos textos de Psicologia Ambiental, os métodos são classificados de maneira ortodoxamente genérica. Assim, Aragonés e Amérigo (1998) afirmam que a maioria dos métodos empregados em Psicologia Ambiental são equivalentes aos da psicologia em geral, a saber: método experimental, experimentos de campo, e estudos correlacionais, aos quais acrescentam "técnicas de investigação" referindo a outros autores. Por seu lado, Bechtel (1997, p. 96) sugere que haveria uma evolução e convergência nos métodos da Psicologia Ambiental (na América do Norte) que vai de muito poucos a muitos estudos empíricos e de um uso de estatística não paramétrica (qui quadrado, especialmente) a um uso de técnicas multivariadas de maior sofisticação.

A Psicologia Ambiental como campo interdisciplinar atraiu, desde as suas origens, especialistas de diferentes disciplinas que tentaram combinar objetivos teóricos e práticos, motivo pelo qual os métodos em Psicologia Ambiental são muito diversos (Holahan, 1991). Considerados globalmente, contudo, não parece haver nenhuma especificidade nos métodos em Psicologia Ambiental com respeito aos da Psicologia em geral. Em nosso entender, tampouco se observa uma diferenciação entre eles associada à periodização das três etapas no desenvolvimento da Psicologia Ambiental (européia) proposta por Pol (2001): arquitetônica ou do habitat (1960-70), da sustentabilidade (1980-90), e a da globalização, atualmente.

Não parece, igualmente, ter sido sugerido que devemos mudar o método científico tradicional por outro distinto dele, não obstante se tenham levantado críticas (de uma perspectiva construtivista) contra a perspectiva positivista em Psicologia Ambiental, cujos trabalhos "não incidiram de maneira significativa na explicação da problemática abordada, por não se terem traduzido em ações, não transcenderem, com a freqüência esperada, para outras disciplinas, âmbitos e atores..." (Wiesenfeld, Sanchez, \& Cronik, 2002, p. 381). É necessário lembrar, contudo, que a ciência vai explicando gradualmente (por acumulação) o seu objeto e isto, naturalmente, está primariamente no nível das idéias, não da ação. 
Em Psicologia Ambiental, atualmente, segue-se utilizando, e aparentemente com êxito (senão já teria se extinguido), métodos "tradicionais", o que não é em absoluto surpreendente já que se trata de métodos científicos cuja essência é a mesma. Assim, por exemplo, ao se tratar da etapa um - psicologia arquitetônica ou urbanística - em que necessitávamos avaliar a satisfação residencial, isto se fazia - e ainda hoje se faz - com desenhos transsecionais descritivos ou explicativos e com escalas ad-hoc do tipo Likert ou com entrevistas individuais ou em profundidade. Atualmente, procede-se de igual maneira ao efetuarmos avaliações de impacto ou indagarmos sobre a identidade social em populações de extrema pobreza com ou sem intervenção do Estado etc. (Moyano Díaz \& Lazcano, 2001) ou (etapas 2 - sustentabilidade, e 3 - globalização) ao estudarmos o comportamento pró-ambiental de reutilização e de reciclagem (Corral-Verdugo, 2002) ou outros fenômenos. Pode ser que nos estudos em Psicologia Ambiental seja mais fácil distinguir ou encontrar uma evolução na análise dos dados (quer dizer, nas técnicas) do que nos próprios métodos. As técnicas vão desde descrições narrativas "a-nexatas", passando por uma estatística convencional uni- ou multivariada, até refinadas técnicas de análise linear estrutural, utilizadas atualmente.

Moore (1991, p. 1390), avaliando os métodos em Psicologia Ambiental, estabelece que tinham sido registrados dois avanços significativos: a) a adoção de métodos de campo quasi experimentais para um amplo leque de fatos, aproximando-se então do controle experimental, de modo consistente, com validez ecológica e, b) um tratamento renovado e sério dos métodos qualitativos de investigação e análise. O primeiro talvez se aplique convenientemente à Psicologia Ambiental na América Latina do século XX, devido principalmente à sua expansão, mas quanto ao segundo isso não é tão evidente já que, se bem que exista interesse pelos métodos qualitativos, estes permanecem em um nível relativamente pouco formalizado de desenvolvimento científico na maior parte dos países da América Latina, segundo avaliamos em outro lugar (Moyano Díaz, 1994a). Os métodos são produtos culturais úteis para buscar respostas a um problema, donde o ponto central é sua eficácia e sua eficiência (cada método tem sua utilidade e valor); contu- 
do, a questão sobre a sua valoração não pode ser apriorística mas, e se assim desejarmos, empírica: verificar se a sua adequação corresponde ao tipo de problemas metodológicos ou empíricos (Moyano Díaz, 1994b). A metodologia em Psicologia Ambiental adota formas variadas e parece poder ser caracterizado - como a Psicologia atual no mundo - por um pluralismo metodológico ou por um multimétodo.

\section{A interdisciplinaridade da Psicologia Ambiental e as possibilidade da intervenção ambiental}

Afirmamos a existência de uma continuidade nos métodos e de uma evolução para uma complexidade crescente nas técnicas. Cada vez mais utilizam-se dispositivos experimentais de investigação e, mais especificamente, quasi-experimentais (Stanley \& Campbell, 1970), os conceitos de confiabilidade e validez, e instrumentos como a amostragem $\mathrm{e}_{2}$ questionários; escalas de atitudes; diferenciais semânticos; e outros foram se popularizando.

Em Psicologia, criaram-se inúmeras teorias de personalidade que permitem descrever e diferenciar pessoas e, em sua aplicação à Psicologia Ambiental, Mckechnie (1978) pode diferenciar oito tipos de personalidades segundo sua disposição ao ambiente, mediante o Inventário de Resposta Ambiental (ERI). Em um enfoque de "personologia ambiental" mais atual sugeriu-se o desenvolvimento de uma metodologia que opere no sentido inverso - desde o comportamento ambiental até os traços de personalidade (Little, 1991), mas isto ainda constitui um desafio. Esta concentração histórica no "intrapsíquico" representou uma deterioração do estudo do componente A (ambiente) e não facilita a comunicação interdisciplinar nem a intervenção ambiental. Não dispomos em nossa disciplina de um cardápio atrativo ou variado de taxonomias do ambiente, sendo estas escassas e não suficientemente consensuais, motivo pelo qual não é fácil categorizar o ambiente para propósitos de descrição ou intervenção, o que representa uma debilidade na hora de interagir com outros profissionais do ambiente. 
Moore (1991) propõe três níveis de análise para estudar o ambiente: a escala espacial do ambiente, a taxonomia de tipos de ambiente e as dimensões ou variáveis ambientais. Em nosso trabalho de avaliação da satisfação residencial em conjuntos residenciais de casas utilizamos, com resultado satisfatório, a escala espacial de Bronfenbrenner (1987) - Macrosistema, Mesosistema, Exosistema e Microsistema - adaptada como Bairro, Conjunto habitacional, Vizinhos e Moradia respectivamente, e estabelecemos, mediante análise de qui-quadrado, dois ńveis diferentes de associação entre os três últimos constructos (Moyano Díaz, 1994a). Em outras pesquisas, segmentamos (taxonomia) o ambiente, segundo o seu pertencimento ou propriedade, em público ou privado, entendendo que o funcionamento, controle e ações de melhoria e de deterioração das pessoas sobre ele é diferente em um e no outro (Moyano Díaz, 1999). Neste mesmo trabalho, referido ao comportamento espacial de melhoramento do entorno imediato da moradia social e inspirados na classificação em três dimensões dos grupos sociais de Moos (1979), medimos três características de funcionamento dos grupos humanos residenciais: relações interpessoais, percepção de funcionalidade do espaço e percepção de possibilidade de intervenção (Moyano Díaz, 1994a).

Necessitamos mais conceitos e modos de segmentação do ambiente e de suas dimensões para facilitar abordagens mais diretas do trabalho em e com o ambiente em equipes interdisciplinares. Na medida em que dispusermos disto poderemos visualizar não apenas mais facilmente nosso papel em equipes constituídas por profissionais da Arquitetura, do Urbanismo, da Geografia e, eventualmente, da Engenharia e da Ecologia, mas também o destes profissionais nessas equipes, tendo assim alguma probabilidade de assumir um papel de condução ou, talvez, de integração.

Parafraseando Gibson, Ivancevich e Donnelly (2001, p. 509), pode-se definir a intervenção ambiental como uma ação ou programa levado a cabo para dirigir o processo de mudança ambiental com um objetivo específico. Tal como assinala Corraliza (1990, p. 22), não existe uma metodologia de intervenção que assegure o rendimento e a efetividade de um programa dado, embora exista a metodologia científica que permite estabelecer relações entre condições antecedentes e suas conseqüências. Seguindo Zube (1984), 
Corraliza reproduz um marco de referência para a intervenção em três dimensões: a ambiental (problema, indicadores e critérios, ambiente percebido vs. real), a institucional (recursos disponíveis, estratégias, metas e objetivos, avaliações prévias) e os participantes (características individuais, de grupo, papéis, usuários, sistemas de crenças). A avaliação dos resultados de qualquer intervenção parece ser uma condição imprescindível quanto à sua efetividade. Quase sempre há aqui, envolvidos e entrelaçados, aspectos propriamente técnicos e aspectos políticos ou sociais.

Uma formação sólida em metodologia científica é um instrumento poderoso para desenhar intervenções ambientais apropriadas. $\mathrm{O}$ uso de dispositivos de observação experimental e, muito especialmente, não experimental sob suas distintas formas (quasi-experimental descritivo ou explicativo, séries cronológicas interrompidas etc.), é uma ferramenta indispensável para elaborar estratégias de intervenção e mecanismos de avaliação de seu seguimento. Um adequado manejo conceptual da confiabilidade e validade dos dispositivos e medidas, assim como das técnicas estatísticas para a análise são competências altamente desejáveis, estando no mesmo nível de uma adequada capacidade de negociação e manejo de conflitos sociais, dado que, em toda intervenção ambiental que devemos enfrentar, há sempre interesses contrapostos ou algum grau de disfuncionalidade social.

\section{Intervenção ambiental e trabalho interdisciplinar}

A intervenção ambiental é, de um ponto de vista geral, uma intervenção marcada pela intervenção social. Quem historicamente, no Chile, tem se ocupado dos problemas sociais das pessoas e de suas famílias, ou a dimensão "participante" nos programas de intervenção ambiental acima referidos, foram os propriamente chamados Assistentes Sociais. Algumas discussões internas destes com respeito ao seu papel como profissionais na sociedade atual mostram, segundo Escortín Caparrós (1992, p. 24), pelo menos três alternativas de resposta à pergunta acerca de como entender "a intervenção social com os clientes, finalidade primordial da prática dos trabalhadores so- 
ciais". Primeira: a prática do trabalho social como resposta aos conflitos de necessidade em indivíduos, grupos e comunidades, quer dizer, ante as insatisfações e malestar produzidos pela carência de satisfação das necessidades humanas básicas. Segundo: o trabalho social como processo para resolver problemas que as pessoas, grupos ou comunidades não podem desbloquear ou resolver. Terceira: a intervenção nas relações transacionais, entendida como a influência para a mudança destas como foco ou objetivo central da intervenção, aponta para a modificação de relações transacionais disfuncionais de indivíduos entre si, ou entre indivíduos e grupos ou com instituições.

A mudança na prática do trabalho social dos anos 50-60 até hoje consistiria em focalizar-se atualmente, não os aspectos intrapessoais (intrapsíquicos) da vida dos clientes, mas os papéis, relações e interações, e assim prestar muito mais atenção do que antes nos fatores ambientais e em sua importância na vida do cliente (Escortín Caparrós, 1992, p. 26).

Sem dúvida, hoje muitos psicólogos jovens se sentem motivados pelo trabalho social implicado na denominada Psicologia Comunitária. Há neles uma sensibilidade com relação à justiça social e, em muitos casos, sua tarefa se superpõe ou confunde com o que Escortín Caparrós denominou acima como próprio aos assistentes sociais o que, sem dúvida, abre interrogações relativas ao papel profissional. As populações, bairros e comunidades hoje apresentam uma maioria de problemas relativos a questões ambientais em que a sua participação informada e educada, unida à ação do Estado, vem sendo cada vez mais necessárias, no caso, para o Chile: (in)segurança do cidadão, a (des)contaminação, a carência de área verdes e espaços públicos, os depósitos de lixo, a morbimortalidade por acidentes de trânsito e de outras causas, etc.

Moyano Díaz, E. (2005). An exploration of methodological specificity and interdisciplinarity in environmental psychology. Psicologia USP, 16(1/2), 141-154. 


\begin{abstract}
This article proposes an exploration and explanation of methodological specificity and interdisciplinarity in Environmental Psychology. To achieve this, it relies on existing typologies referring to the types of problems, investigations and methodological approaches. It concludes that there is a methodological pluralism in Environmental Psychology and that the methods are useful products, even though cultural ones, to search for answers to a problem, provided that they are considered in terms of their efficacy and efficiency, regarding their empirical valuation. Environmental intervention is, in turn, marked by social intervention.
\end{abstract}

Index terms: Interdisciplinary research. Methodology. Enviromental psychology.

Moyano Diaz, E. (2005). Une exploration de la specificite et interdisciplinarite methodologique en psychologie environnementale. Psicologia Usp, 16(1/2), 141-154.

Résumé: Cet article propose une exploration et une explicitation de la spécificité et de l'interdisciplinarité méthodologique en Psychologie Environnementale. Pour ce faire, il se base sur des typologies existantes concernant les types de problèmes, d'investigations et d'optiques méthodologiques. Il en conclut que les méthodes sont des produits culturels utiles pour la recherche de réponses à un problème, du point de vue de leur efficacité et de leur efficience, avec un type d'estimation empirique, et que dans la Psychologie Environnementale, advient un pluralisme méthodologique. L'intervention environnementale est, de son côté, marquée par l'intervention sociale.
Mots-Clés: $\quad$ Interdisciplinarité.
Méthodologie. Psychologie environnementale.

\title{
Referências
}

Altman, I., \& Rogoff, B. (1991). World views in psychology: Trait, interactional, organismic, and transactional perspectives. In D. Stokols \& I. Altman (Eds.), Handbook of environmental psychology (Vol. 1, pp. 7-40). Malabar, Florida: Krieger Pub. 
Aragonés, J. I., \& Amérigo, M. (1998). Psicología ambiental. Madrid: Pirámide.

Bechtel, R. B. (1997). Environment and behavior. An introduction. New York: Sage.

Bronfenbrenner, U. (1987). La ecología del desarrollo humano. Barcelona: Paidós.

Bunge, M. (1992). La ciencia su método y su filosofía. Buenos Aires: Siglo Veinte.

Bunge, M., \& Ardila, R. (1988). Filosofía de la psicología. Barcelona: Ariel.

Corral-Verdugo, V. (2002). Avances y limitaciones en la medición del comportamiento pro-ambiental. In J. Guevara \& S. Mercado (Eds.), Temas selectos en psicología ambiental (pp. 483-510). México: UNAM / GRECO / Fundación UNILIBRE. (483510.)

Corraliza, J. A. (1990). Metodología de los programas de intervención ambiental. In R. De Castro, J. I. Aragonés, \& J. A. Corraliza (Eds.), La conservación del entorno. Programas de intervención en psicología ambiental. Sevilla, España: Junta de Andalucía, Agencia del Medio Ambiente.

De Ketele, J. M., \& Roegiers, X. (1993). Methodologie du recueil d'informations. Belgium: Ed. Pedagogies en Dévelopment, De Boeck Université.

Escartín Caparrós, M. J. (1992). Manual de trabajo social (modelos de práctica profesional) (Colección Amalgama). Alicante, España: Editorial Aguaclara.

Gibson, J. L., Ivancevich, J. M., \& Donnelly, J. H. (2001). Las organizaciones. Comportamiento, estructura, procesos. Santiago: McGraw Hill Interamericana.

Hernández S., R., Fernández, C., \& Baptista L. P. (1993). Metodología de la investigación. New York: McGraw Hill.

Holahan, C. J. (1991). Psicología ambiental. Un enfoque integral. México: Limusa.

Little, B. R. (1991). Personality and the environment. In D. Stokols, \& I. Altman (Eds.), Handbook of environmental psychology (Vol. 1, Part 2, Chap. 7, pp. 205-244). Malabar, FL: Krieger Pub.

Mckechnie, G. E. (1978). Environmental dispositions: Concepts and mesures. In P. McReynolds (Ed.), Advances in psychological assessment (Vol. 4). San Francisco, CA: J. Bass.

Moore, G. T. (1991). Environment and behavior research in North America: History, developments, and unresolved issues. In D. Stokols, \& I. Altman (Eds.), Handbook of environmental psychology (Vol. 2, Chapter 39, pp. 1359-1410). Malabar, FL: Krieger Pub.

Moos, R. (1979). Evaluating educational environments. San Francisco, CA: Jossey Bass. 
Moyano Díaz, E. (1994a). Diseño habitacional y comportamiento espacial de mejoramiento y deterioro en sectores de vivienda social. In E. Wiesenfeld (Coord.), Contribuciones iberoamericanas a la psicología ambiental. Caracas: UCV.

Moyano Díaz, E. (1994b). Hacia la integración de metodologías cualitativas y cuantitativas en la investigación en ciencias sociales. Revista de la Facultad de Humanidades, 4, 59-86.

Moyano Díaz, E. (1999). Psicología ambiental. Estudios y aplicaciones. Santiago, Chile: Facultad de Humanidades y Es cuela de Psicología, Universidad de Santiago de Chile.

Moyano Díaz, E., \& Lazcano, M. (2001). Exploración de la identidad social urbana en campamentos de extrema pobreza. Revista $A+C$, 1. Escuela de Arquitectura, Universidad de Santiago de Chile.

Pol, E. (2001). Ejes de tensión y nueva agenda para la psicología ambiental. Una perspectiva europea. In E. Tassara (Coord.), Panoramas interdisciplinares para uma psicologia ambiental do urbano. São Paulo: EDUC / FAPESP.

Sierra Bravo, R. (1987). Técnicas de investigación social. Teoría y ejercicios. Madrid: Paraninfo.

Stanley, J., \& Campbell, D. (1970). Diseños experimentales y cuasiexperimentales en la investigación social. Buenos Aires: Amorrortu.

Wiesenfeld, E., Sánchez, E., \& Cronik K. (2002). La intervención ambiental participativa: fundamentos y aplicaciones. In J. Guevara, \& S. Mercado (Coords.), Temas selectos en psicología ambiental. (2002). México: UNAM / GRECO / Fundación UNILIBRE. (377-410).

Recebido em 5.04.2004

Revisto e encaminhado em 23.02.2005

Aceito em: 7.03.2005 